\title{
ON THE PROBLEM OF DETECTION OF ISOLATED BLACK HOLES
}

\author{
V. F. SHVARTSMAN
}

Special Astrophysical Observatory, U.S.S.R. Academy of Sciences, St. Zelenchukskaya, Stravropolsky Kraj, 357140 U.S.S.R.

\begin{abstract}
The interstellar plasma accreted by isolated black holes should be heated during the infall process and radiate in the magnetic fields connected with the infalling material. A portion of the radiation in the optical range is likely to have a luminosity of order $10^{27}-10^{30} \mathrm{erg} \mathrm{s}^{-1}$. In $S A O$ U.S.S.R. $A S$ an experiment is being carried out to observe isolated black holes as faint optical stars without spectral lines. So far the results of the experiment are negative.
\end{abstract}

\section{References}

Shvartsman, V. F.: 1970, preprint, IAM 42, Soviet Astron. AJ 15, 377, 1971.

Shvartsman, V. F.: 1973, Commun. SAO U.S.S.R. AS, in press. 